\title{
Properties of the Sobolev space $H_{k}^{s, s^{\prime}}$
}

\author{
by Henryk KoŁakowski (Warszawa)
}

Abstract. Let $n \geq 2$ and $H_{k}^{s, s^{\prime}}=\left\{u \in S^{\prime}\left(\mathbb{R}^{n}\right):\|u\|_{s, s^{\prime}}<\infty\right\}$, where

$$
\|u\|_{s, s^{\prime}}^{2}=(2 \pi)^{-n} \int\left(1+|\xi|^{2}\right)^{s}\left(1+\left|\xi^{\prime}\right|^{2}\right)^{s^{\prime}}|F u(\xi)|^{2} d \xi,
$$

$F u(\xi)=\int e^{-i x \xi} u(x) d x, \xi^{\prime} \in \mathbb{R}^{k}, k<n$. We prove that for some $s, s^{\prime}$ the space $H_{k}^{s, s^{\prime}}$ is a multiplicative algebra.

Let $n \geq 2$ and $H_{k}^{s, s^{\prime}}=\left\{u \in S^{\prime}\left(\mathbb{R}^{n}\right):\|u\|_{s, s^{\prime}}<\infty\right\}$, where

$$
\|u\|_{s, s^{\prime}}^{2}=(2 \pi)^{-n} \int\left(1+|\xi|^{2}\right)^{s}\left(1+\left|\xi^{\prime}\right|^{2}\right)^{s^{\prime}}|F u(\xi)|^{2} d \xi,
$$

$\widehat{u}(\xi)=F u(\xi)=\int e^{-i x \xi} u(x) d x, \xi^{\prime}=\left(\xi_{1}, \ldots, \xi_{k}\right), k<n$. Below we write $H^{s, s^{\prime}}$ instead of $H_{k}^{s, s^{\prime}}$.

This note contains the proof of the following theorem:

If $s>(n-k) / 2, s+s^{\prime}>n / 2$ and $s+2 s^{\prime}>(n-k) / 2$, then the space $H^{s, s^{\prime}}$ is a multiplicative algebra over $\mathbb{C}$.

A large part of calculations is similar of those of M. Sable-Tougeron [1] for $k=n-1$.

We expect that the above theorem will be an effective tool for further research of the properties of solutions to nonlinear elliptic equations with boundary value conditions on submanifolds of arbitrary codimension (for linear equations see [2]).

Let $\xi=\left(\xi^{\prime}, \xi^{\prime \prime}\right), \xi^{\prime \prime}=\left(\xi_{k+1}, \ldots, \xi_{n}\right),|\xi|=\left(\xi_{1}^{2}+\ldots+\xi_{n}^{2}\right)^{1 / 2}, \phi \in C_{0}^{\infty}\left(\mathbb{R}^{n}\right)$,

$$
\phi(\xi)= \begin{cases}1 & \text { for }|\xi| \leq 1 / 2 \\ 0 & \text { for }|\xi| \geq 1\end{cases}
$$

$\phi(\xi)=\phi(|\xi|) \geq 0, \phi^{\prime}\left(\xi^{\prime}\right)=\phi\left(\xi^{\prime}, 0\right)$, and $u \in S^{\prime}\left(\mathbb{R}^{n}\right)$. For $p, p^{\prime} \in \mathbb{N}$ we define

1991 Mathematics Subject Classification: Primary 46E35.

Key words and phrases: multiplicative algebra, Littlewood double decomposition. 
the operators $S_{p}$ and $S_{p^{\prime}}$ by

$$
F\left(S_{p} u\right)(\xi)=\phi\left(2^{-p} \xi\right) F u(\xi), \quad F\left(S_{p^{\prime}}^{\prime} u\right)(\xi)=\phi^{\prime}\left(2^{-p^{\prime}} \xi^{\prime}\right) F u(\xi) .
$$

We use the following notations:

$$
\begin{aligned}
S_{p p^{\prime}} & =S_{p} \circ S_{p^{\prime}}, & \Delta_{p^{\prime}} & =S_{p^{\prime}+1}^{\prime}-S_{p^{\prime}}^{\prime} \\
\Delta_{p} & =S_{p+1}-S_{p}, & \Delta_{p p^{\prime}} & =\Delta_{p} \circ \Delta_{p^{\prime}}^{\prime} .
\end{aligned}
$$

It is easy to see that

$$
F\left(\Delta_{p} u\right)(\xi)=\psi\left(2^{-p} \xi\right) F u(\xi), \quad F\left(\Delta_{p^{\prime}}^{\prime} u\right)(\xi)=\psi^{\prime}\left(2^{-p^{\prime}} \xi^{\prime}\right) F u(\xi),
$$

where

$$
\psi(\xi)=\phi(\xi / 2)-\phi(\xi), \quad \psi^{\prime}\left(\xi^{\prime}\right)=\psi\left(\xi^{\prime}, 0\right) .
$$

Of course

$$
\phi(\xi)+[\phi(\xi / 2)-\phi(\xi)]+[\phi(\xi / 4)-\phi(\xi / 2)]+\ldots=\phi(\xi)+\sum_{p \geq 0} \psi\left(2^{-p} \xi\right)=1 .
$$

Similarly

$$
\phi^{\prime}\left(\xi^{\prime}\right)+\sum_{p^{\prime} \geq 0} \psi^{\prime}\left(2^{-p^{\prime}} \xi^{\prime}\right)=1 .
$$

The following formula (the Littlewood double decomposition) is true:

$$
u=S_{00} u+S_{0} \Delta_{0}^{\prime} u+\sum_{p \geq 0} \Delta_{p} S_{0}^{\prime} u+\sum_{p, p^{\prime} \geq 0} \Delta_{p p^{\prime}} u .
$$

Indeed,

$$
\begin{aligned}
F u= & \left(\phi(\xi) \phi^{\prime}\left(\xi^{\prime}\right)+\phi(\xi) \psi^{\prime}\left(\xi^{\prime}\right)\right. \\
& \left.+\sum_{p \geq 0} \psi\left(2^{-p} \xi\right) \phi^{\prime}\left(\xi^{\prime}\right)+\sum_{p, p^{\prime} \geq 0} \psi\left(2^{-p} \xi\right) \psi^{\prime}\left(2^{-p^{\prime}} \xi^{\prime}\right)\right) F u \\
= & \left(\phi(\xi)+\sum_{p \geq 0} \psi\left(2^{-p} \xi\right)\right)\left(\phi^{\prime}\left(\xi^{\prime}\right)+\sum_{p^{\prime} \geq 0} \psi^{\prime}\left(2^{-p^{\prime}} \xi^{\prime}\right)\right) F u .
\end{aligned}
$$

REMARK. We have

$$
\sum_{p^{\prime} \geq 0} \phi(\xi) \psi^{\prime}\left(2^{-p^{\prime}} \xi^{\prime}\right)=\phi(\xi) \psi\left(\xi^{\prime}\right)
$$

In the component $\sum_{p, p^{\prime} \geq 0} \psi\left(2^{-p} \xi\right) \psi^{\prime}\left(2^{-p^{\prime}} \xi^{\prime}\right)$ only elements with $p^{\prime} \geq p+1$ are important. Moreover $S_{00}+S_{0} \Delta_{0}^{\prime}=S_{01}$.

TheOREM 1 (Characterization of the space $H^{s, s^{\prime}}$ ). A function $u$ belongs to $H^{s, s^{\prime}}$ if and only if

$$
\left\|S_{01} u\right\|_{0}^{2}+\sum_{p \geq 0} 4^{p s}\left\|\Delta_{p} S_{0}^{\prime} u\right\|^{2}+\sum_{p, p^{\prime} \geq 0} 4^{p s+p^{\prime} s^{\prime}}\left\|\Delta_{p p^{\prime}} u\right\|_{0}^{2}<\infty .
$$


Proof. Let $u \in H^{s, s^{\prime}}$. Denote by $\kappa_{r}$ and $\kappa_{\phi}$ the characteristic functions of $\operatorname{supp} \psi\left(2^{-r}\right.$. $)$ and $\operatorname{supp} \phi$ respectively. On $\operatorname{supp} \psi\left(2^{-r}\right.$.) we have $2^{r} \approx|\xi|$. It is easy to see that

$$
\begin{aligned}
\phi^{2}(\xi) & \phi^{\prime 2}\left(\xi^{\prime} / 2\right) \\
& +\sum_{p \geq 0} 4^{p s} \psi^{2}\left(2^{-p} \xi\right) \phi^{\prime 2}\left(\xi^{\prime}\right)+\sum_{p, p^{\prime} \geq 0} 4^{p s+p^{\prime} s^{\prime}} \psi^{2}\left(2^{-p} \xi\right) \phi^{\prime 2}\left(2^{-p^{\prime}} \xi^{\prime}\right) \\
\leq & c_{1}\left(\kappa_{\phi}(\xi)+\sum_{p \geq 0} \kappa_{p}(\xi)\left(|\xi|^{2}\right)^{s}+\sum_{p, p^{\prime} \geq 0} \kappa_{p}(\xi) \kappa_{p^{\prime}}\left(\xi^{\prime}\right)\left(|\xi|^{2}\right)^{s}\left(\left|\xi^{\prime}\right|^{2}\right)^{s^{\prime}}\right) \\
\leq & c_{2}\left(1+\sum_{p \geq 0} \kappa_{p}(\xi)\left(|\xi|^{2}\right)^{s}\right)\left(1+\sum_{p^{\prime} \geq 0} \kappa_{p^{\prime}}\left(\xi^{\prime}\right)\left(\left|\xi^{\prime}\right|^{2}\right)^{s^{\prime}}\right) \\
\leq & c\left(1+|\xi|^{2}\right)^{s}\left(1+\left|\xi^{\prime}\right|^{2}\right)^{s^{\prime}} .
\end{aligned}
$$

Multiplying by $|\widehat{u}(\xi)|^{2}$ and integrating with respect to $\xi$ we get inequality (1).

The second part of the proof is contained in the following lemmas.

Lemma 1. If $\left\{u_{p p^{\prime}}\right\}_{p, p^{\prime} \geq 0} \subset L^{2}, \operatorname{supp} F u_{p p^{\prime}}$ is in the 2-ring

$$
\left\{\gamma^{-1} 2^{p+q} \leq|\xi| \leq \gamma 2^{p+q} ; \gamma^{-1} 2^{p^{\prime}+q^{\prime}} \leq\left|\xi^{\prime}\right| \leq \gamma 2^{p^{\prime}+q^{\prime}}\right\}
$$

with $\gamma>, q, q^{\prime} \geq 0$, and $\sum_{p, p^{\prime} \geq 0} 4^{p s+p^{\prime} s^{\prime}}\left\|u_{p p^{\prime}}\right\|_{0}^{2}<\infty$, then $u=\sum_{p, p^{\prime} \geq 0} u_{p p^{\prime}}$ belongs to $H^{s, s^{\prime}}$, and

$$
\|u\|_{s, s^{\prime}} \leq C\left(\sum_{p, p^{\prime} \geq 0} 4^{(p+q) s+\left(p^{\prime}+q^{\prime}\right) s^{\prime}}\left\|u_{p p^{\prime}}\right\|_{0}^{2}\right)^{1 / 2},
$$

where $C$ depends only on $\gamma, s$ and $s^{\prime}$.

Lemma 2. If $\left\{u_{p}\right\}_{p \geq 0} \subset L^{2}, \operatorname{supp} F u_{p}$ is in the set

$$
\Omega=\left\{\gamma^{-1} 2^{p} \leq|\xi| \leq \gamma 2^{p} ;\left|\xi^{\prime}\right| \leq \gamma^{\prime}\right\}
$$

with constants $\gamma>1, \gamma^{\prime}>0$ and $\sum_{p \geq 0} 4^{p s}\left\|u_{p}\right\|_{0}^{2}<\infty$, then $u=\sum_{p \geq 0} u_{p} \in$ $H^{s, \infty}=\bigcap_{s^{\prime} \in \mathbb{R}} H^{s, s^{\prime}}$, and

$$
\|u\|_{s, s^{\prime}} \leq C\left\{\sum_{p \geq 0} 4^{p s}\left\|u_{p}\right\|_{0}^{2}\right\}^{1 / 2}
$$

where the constant $C$ depends only on $\gamma, \gamma^{\prime}, s$ and $s^{\prime}$. If additionally $s>0$, then instead of $\Omega$ one can take the set $\left\{|\xi| \leq \gamma 2^{p} ;\left|\xi^{\prime}\right| \leq \gamma^{\prime}\right\}$.

Proof. Observe that for fixed $\xi, F u_{p p^{\prime}}(\xi) \neq 0$ and $F u_{p}(\xi) \neq 0$ only for a finite number of functions, and that the number depends only on $\gamma$ and $\gamma^{\prime}$. 
Hence, there exists a constant $C=C\left(\gamma, \gamma^{\prime}\right)$ such that

$$
\begin{aligned}
\forall \xi \in \mathbb{R}^{n}\left|\sum_{p, p^{\prime} \geq 0} F u_{p p^{\prime}}(\xi)\right|^{2} \leq C \sum_{p, p^{\prime} \geq 0}\left|F u_{p p^{\prime}}(\xi)\right|^{2} \quad \text { (for Lemma 1) }, \\
\left|\sum_{p \geq 0} F u_{p}(\xi)\right|^{2} \leq C \sum_{p \geq 0}\left|F u_{p}(\xi)\right|^{2} \quad \text { (for Lemma 2). }
\end{aligned}
$$

Multiplying by $\left(1+|\xi|^{2}\right)^{s}\left(1+\left|\xi^{\prime}\right|^{2}\right)^{s^{\prime}}$ and integrating over $\mathbb{R}^{n}$ we get the assertion of the two lemmas.

For the 2-ball $\left\{|\xi| \leq \gamma 2^{p} ;\left|\xi^{\prime}\right| \leq \gamma^{\prime}\right\}$ we write

$$
F u=\left(\phi(\xi)+\sum_{r \geq 0} \psi\left(2^{-r} \xi\right)\right) \sum_{p \geq 0} F u_{p}
$$

This means that $u=S_{0} \sum_{p \geq 0} u_{p}+\sum_{r \geq 0} \Delta_{r} \sum_{r \leq p+N} u_{p}$ with a suitable $N$. According to assumption $2^{p s}\left\|u_{p}\right\|_{0} \leq \eta_{p}, \sum_{p \geq 0} \eta_{p}^{2}<\infty$. We have

$$
\left\|\Delta_{r} \sum_{r \leq p+N} u_{p}\right\|_{0} \leq \sum_{r \leq p+N} 2^{-p s} \eta_{p} \leq c 2^{-r s} \eta_{r},
$$

where the constant $c$ does not depend on $r, s$, hence $u \in H^{s, s^{\prime}}$.

For $s$ or $s^{\prime}$ positive one can prove the following statements:

Lemma 3. If $s>0,\left\{u_{p p^{\prime}}\right\}_{p, p^{\prime} \geq 0} \subset L^{2}$,

$$
\operatorname{supp} F u_{p p^{\prime}} \subset\left\{|\xi| \leq \gamma 2^{p} ; \gamma^{-1} 2^{p^{\prime}+q^{\prime}} \leq\left|\xi^{\prime}\right| \leq \gamma 2^{p^{\prime}+q^{\prime}}\right\}
$$

with $\gamma>1, q^{\prime} \geq 0$, and $\sum_{p, p^{\prime} \geq 0} 4^{p s+p^{\prime} s^{\prime}}\left\|u_{p p^{\prime}}\right\|_{0}^{2}<\infty$, then $u=\sum_{p, p^{\prime} \geq 0} u_{p p^{\prime}}$ $\in H^{s, s^{\prime}}$, and

$$
\|u\|_{s, s^{\prime}} \leq C\left(\sum_{p, p^{\prime} \geq 0} 4^{p s+\left(p^{\prime}+q^{\prime}\right) s^{\prime}}\left\|u_{p p^{\prime}}\right\|_{0}^{2}\right)^{1 / 2},
$$

where $C$ depends only on $\gamma, s$ and $s^{\prime}$.

LEMma 4. If $s^{\prime}>0,\left\{u_{p p^{\prime}}\right\}_{p, p^{\prime} \geq 0} \subset L^{2}$,

$$
\operatorname{supp} F u_{p p^{\prime}} \subset\left\{\gamma^{-1} 2^{p+q} \leq|\xi| \leq \gamma 2^{p+q} ;\left|\xi^{\prime}\right| \leq \gamma 2^{p^{\prime}}\right\}
$$

with $\gamma>1, q \geq 0$, and $\sum_{p, p^{\prime} \geq 0} 4^{p s+p^{\prime} s^{\prime}}\left\|u_{p p^{\prime}}\right\|_{0}^{2}<\infty$, then $u=\sum_{p, p^{\prime} \geq 0} u_{p p^{\prime}}$ $\in H^{s, s^{\prime}}$, and

$$
\|u\|_{s, s^{\prime}} \leq C\left(\sum_{p, p^{\prime} \geq 0} 4^{(p+q) s+p^{\prime} s^{\prime}}\left\|u_{p p^{\prime}}\right\|_{0}^{2}\right)^{1 / 2},
$$

where $C$ depends only on $\gamma, s$ and $s^{\prime}$.

Lemma 5. If $s>0, s^{\prime}>0,\left\{u_{p p^{\prime}}\right\}_{p, p^{\prime} \geq 0} \subset L^{2}$,

$$
\operatorname{supp} F u_{p, p^{\prime}} \subset\left\{|\xi| \leq \gamma 2^{p} ;\left|\xi^{\prime}\right| \leq \gamma 2^{p^{\prime}}\right\}
$$


with $\gamma>1$, and $\sum_{p, p^{\prime} \geq 0} 4^{p s+p^{\prime} s^{\prime}}\left\|u_{p p^{\prime}}\right\|_{0}^{2}<\infty$, then $u=\sum_{p, p^{\prime} \geq 0} u_{p p^{\prime}} \in$ $H^{s, s^{\prime}}$, and

$$
\|u\|_{s, s^{\prime}} \leq C\left(\sum_{p, p^{\prime} \geq 0} 4^{p s+p^{\prime} s^{\prime}}\left\|u_{p p^{\prime}}\right\|_{0}^{2}\right)^{1 / 2},
$$

where $C$ depends only on $\gamma, s$ and $s^{\prime}$.

Proof of Lemma 3. According to the assumption about $F u_{p p^{\prime}}$ we can write $v_{r p^{\prime}}=\Delta_{r} \sum_{r \leq p+N} u_{p p^{\prime}}$, where $r \geq-1, \Delta_{-1}=S_{0}$, and $N$ does not depend on $\gamma$. Let $\varepsilon_{p p^{\prime}}=2^{p s+p^{\prime} s^{\prime}}\left\|u_{p p^{\prime}}\right\|_{0}$. We have

$$
\begin{aligned}
\left\|v_{r p^{\prime}}\right\|_{0} & \leq c \sum_{r \leq p+N}\left\|u_{p p^{\prime}}\right\|_{0} \leq c \sum_{r \leq p+N} 2^{-p s-p^{\prime} s^{\prime}} \varepsilon_{p p^{\prime}} \\
& =c \sum_{r \leq p+N} 2^{(r-p) s} \varepsilon_{p p^{\prime}} 2^{-r s-p^{\prime} s^{\prime}}=c 2^{-r s-p^{\prime} s^{\prime}} \eta_{r+1, p^{\prime}},
\end{aligned}
$$

where

$$
\eta_{r+1, p^{\prime}}=\sum_{r \leq p+N} 2^{(r-p) s} \varepsilon_{p p^{\prime}} .
$$

We know that $\sum_{p, p^{\prime} \geq 0} 4^{p s+p^{\prime} s^{\prime}}\left\|u_{p p^{\prime}}\right\|_{0}^{2}<\infty, s>0$. Applying the equality

$$
\begin{aligned}
\eta_{r+1, p^{\prime}} & =\sum_{r \leq p+N} \varepsilon_{p p^{\prime}}=\sum_{r \leq p+N} 2^{(r-p) s} 2^{p s+p^{\prime} s^{\prime}}\left\|u_{p p^{\prime}}\right\|_{0} \\
& =\sum_{r \leq p+N} 2^{r s+p^{\prime} s^{\prime}}\left\|u_{p p^{\prime}}\right\|_{0}
\end{aligned}
$$

we can write $\left\{\eta_{r, p^{\prime}}\right\}_{r} \in \ell^{2}(\mathbb{N}),\left\|\left\{\eta_{r, p^{\prime}}\right\}_{r}\right\|_{\ell^{2}(\mathbb{N})} \leq c\left\|\left\{\varepsilon_{p p^{\prime}}\right\}_{p}\right\|_{\ell^{2}(\mathbb{N})}$, where $c$ depends only on $N$ and $s$ (see (2)). By Lemmas 1 and 2, we have $u=$ $\sum_{r \geq-1, p^{\prime} \geq 0} v_{r p^{\prime}} \in H^{s, s^{\prime}}$, and

$$
\begin{aligned}
\|u\|_{s, s^{\prime}} & \leq C\left(\sum_{r, p^{\prime}} 4^{r s+p^{\prime} s^{\prime}+q^{\prime} s^{\prime}-r s-p^{\prime} s^{\prime}} \eta_{r+1, p^{\prime}}^{2}\right)^{1 / 2}=C\left(\sum_{r, p^{\prime}} 4^{q^{\prime} s^{\prime}} \eta_{r+1, p^{\prime}}^{2}\right)^{1 / 2} \\
& \leq C\left(\sum_{p, p^{\prime}} 4^{p s+\left(p^{\prime}+q^{\prime}\right) s^{\prime}}\left\|u_{p p^{\prime}}\right\|_{0}^{2}\right)^{1 / 2} .
\end{aligned}
$$

Proof of Lemma 4. The proof is similar to the proof of Lemma 3. This time one has to study $\Delta_{r}^{\prime} \sum_{p}^{\prime} u_{p p^{\prime}}$ for $r \geq-1$ with $\Delta^{\prime}=S_{0}^{\prime}$.

Proof of Lemma 5. We start with the term $\Delta_{r, r^{\prime}} \sum_{p, p^{\prime}} u_{p p^{\prime}}$ for $r, r^{\prime} \geq-1$ and next we use Lemmas 1 and 2. 
Definition. We write $u \in C^{\alpha}\left(\mathbb{R}^{n}\right), \alpha \in(0,1)$, if $u$ is bounded and

$$
|u(x+y)-u(x)| \leq C|y|^{\alpha} \quad \text { for }|y| \leq 1,
$$

and we write $u \in C_{*}^{1}\left(\mathbb{R}^{n}\right)$ if $u$ is bounded and

$$
|u(x+y)+u(x-y)-2 u(x)| \leq C|y| \quad \text { for }|y| \leq 1 .
$$

If $\varrho>1, \varrho=m+\alpha$, where $m \in \mathbb{N}, \alpha \in(0,1)$, then the space $C^{\varrho}$ is defined to be $\left\{u \in C^{\alpha}: \forall \beta \in \mathbb{N}^{n},|\beta| \leq m, \partial^{\beta} u \in C^{\alpha}\right\}$. If $\varrho=m+1$ then instead of $C^{1}$ we take $C_{*}^{1}$.

Now we prove that for suitable $s, s^{\prime}$ and $\varrho$ the space $H^{s, s^{\prime}}$ is embedded in $C^{\varrho}$.

Theorem 2. If $s>(n-k) / 2, s+s^{\prime}>n / 2$ and $\varrho=\min (s-(n-k) / 2$, $\left.s+s^{\prime}-n / 2\right)$ when $s^{\prime} \neq k / 2$ and $\varrho<s-(n-k) / 2$ when $s^{\prime}=k / 2$, and $u \in H^{s, s^{\prime}}$, then $u \in C^{\varrho}$.

P roof. First we estimate $|u(x+y)-u(x)|$ :

$$
\begin{aligned}
|u(x+y)-u(x)| \leq & (2 \pi)^{-n}\left|\int \widehat{u}(\xi) e^{i x \xi}\left(e^{i y \xi}-1\right) d \xi\right| \\
\leq & C\left(\int|\widehat{u}(\xi)|^{2}(1+|\xi|)^{2 s}\left(1+\left|\xi^{\prime}\right|\right)^{2 s^{\prime}} d \xi\right)^{1 / 2} \\
& \times\left(\int\left|e^{i y \xi}-1\right|^{2}(1+|\xi|)^{-2 s}\left(1+\left|\xi^{\prime}\right|\right)^{-2 s^{\prime}} d \xi\right)^{1 / 2} .
\end{aligned}
$$

Let $|y|<1$. Then

$$
\begin{aligned}
\int\left|e^{i y \xi}-1\right|^{2}(1+|\xi|)^{-2 s}\left(1+\left|\xi^{\prime}\right|\right)^{-2 s^{\prime}} d \xi & \\
\leq & C\left(\int_{|\xi| \leq|y|^{-1}}|y|^{2}|\xi|^{2}(1+|\xi|)^{-2 s}\left(1+\left|\xi^{\prime}\right|\right)^{-2 s^{\prime}} d \xi\right. \\
& \left.+\int_{|\xi| \geq|y|^{-1}}(1+|\xi|)^{-2 s}\left(1+\left|\xi^{\prime}\right|\right)^{-2 s^{\prime}} d \xi\right)=A+B .
\end{aligned}
$$

If we substitute $\xi^{\prime \prime}=\left(1+\left|\xi^{\prime}\right|\right)^{n-k} \eta$, then $d \xi=\left(1+\left|\xi^{\prime}\right|\right)^{n-k} d \xi^{\prime} d \eta,|\xi|^{2}=$ $\left|\xi^{\prime}\right|^{2}+\left(1+\left|\xi^{\prime}\right|\right)^{2}|\eta|^{2} \leq\left(1+\left|\xi^{\prime}\right|^{2}\right)(1+|\eta|)^{2}$ and so

$$
\begin{aligned}
A & \leq c|y|^{2}\left(1+\int_{0}^{|y|^{-1}} \int_{0}^{|y|^{-1}} \frac{r^{k-1} t^{n-k-1} d r d t}{(1+r)^{2 s+2 s^{\prime}-n+k-2}(1+t)^{2 s-2}}\right) \\
& \leq C|y|^{2}\left(1+\int_{1}^{|y|^{-1}} \frac{d r}{r^{2 s+2 s^{\prime}-n-1}} \int_{1}^{|y|^{-1}} \frac{d t}{t^{2 s-n+k-1}}\right) \\
& \leq C|y|^{2}\left(1+\left(|y|^{2 s+2 s^{\prime}-n-2}-1\right)\left(|y|^{2 s-n+k-2}-1\right)\right),
\end{aligned}
$$




$$
\begin{aligned}
B & \leq \int_{\left|\xi^{\prime}\right|>c|y|^{-1},|\eta|>c|y|^{-1}} \frac{\left(1+\left|\xi^{\prime}\right|\right)^{n-k}}{\left(1+\left|\xi^{\prime}\right|\right)^{2 s+2 s^{\prime}}(1+|\eta|)^{2 s}} d \xi^{\prime} d \eta \\
& \leq C \int_{|y|^{-1}}^{\infty} \frac{d r}{r^{2 s+2 s^{\prime}-n-1}} \int_{|y|^{-1}}^{\infty} \frac{d t}{t^{2 s-n+k+1}} \leq c|y|^{2 s+2 s^{\prime}-n}|y|^{2 s-(n-k)} .
\end{aligned}
$$

Similarly we can write

$$
\begin{aligned}
\mid u(x+y)+ & u(x-y)-2 u(x) \mid \\
& \leq C\|u\|_{s, s^{\prime}}\left(\int|\cos x \xi-1|^{2}(1+|\xi|)^{-2 s}\left(1+\left|\xi^{\prime}\right|\right)^{-2 s^{\prime}} d \xi\right)^{1 / 2} .
\end{aligned}
$$

Supposing that $s>(n-k) / 2, s+s^{\prime}>n / 2$ and $\varrho=\min (s-(n-k) / 2$, $\left.s+s^{\prime}-n / 2\right)$ if $s^{\prime} \neq k / 2$ and $\varrho<s-(n-k) / 2$ if $s^{\prime}=k / 2$, we conclude that $u \in C^{Q}$.

Let now $u \in C^{\varrho}, \varrho \in(0,1)$. As $F S_{0} u(\xi)=\phi(\xi) F u(\xi)=\Phi \cdot F u=F(\Phi * u)$ we have

$$
\begin{aligned}
&\left|S_{0} u\right|=|\Phi * u|=\left|\int u(x-t) \Phi(t) d t\right| \leq\|u\|_{L^{\infty}} \int|\Phi(t)| d t<\infty, \\
&\left|\Delta_{p} u(x)\right|=\left|u(t) *\left[2^{n(p+1)} \Phi\left(2^{p+1} t\right)-2^{n p} \Phi\left(2^{p} t\right)\right](x)\right| \\
&=\left|\int\left(u\left(x-t / 2^{p+1}\right)-u\left(x-t / 2^{p}\right)\right) \Phi(t) d t\right| \\
& \leq \int \frac{\left|u\left(x-t / 2^{p+1}\right)-u\left(x-t / 2^{p}\right)\right|}{\left(t / 2^{p}\right)^{\varrho} 2^{-\varrho}}\left(t / 2^{p}\right)^{\varrho} \Phi(t) 2^{-\varrho} d t \leq C 2^{-\varrho p}, \\
& F S_{p} u=\phi\left(2^{-p} \xi\right) F u=F\left(2^{n p} \Phi\left(2^{p} \cdot\right)\right) F u, \quad S_{p} u=2^{n p} \Phi\left(2^{p} \cdot\right) * u, \\
&\left|S_{p} u\right| \leq\|u\|_{L^{\infty}} \int|\Phi(t)| d t=c\|u\|_{L^{\infty}} .
\end{aligned}
$$

From this one obtains $\left|\Delta_{p p^{\prime}} u\right| \leq C 2^{-\varrho p}$, and similarly $\left|\Delta_{p} S_{p}^{\prime} u\right| \leq C 2^{-\varrho p}$. Analogous calculations can be done for $\varrho=1$.

Conclusion. If $u \in C^{\varrho}$, then

$$
\begin{gathered}
\left\|\Delta_{p} u\right\|_{L^{\infty}} \leq C 2^{-\varrho p}, \quad\left\|S_{0} u\right\|_{L^{\infty}} \leq C, \\
\left\|\Delta_{p p^{\prime}} u\right\|_{L^{\infty}} \leq C 2^{-\varrho p} \text { and } \quad\left\|\Delta_{p} S_{p}^{\prime} u\right\|_{L^{\infty}} \leq C 2^{-\varrho p} .
\end{gathered}
$$

Let $u$ and $v$ belong to $H^{s, s^{\prime}}$. Using the Littlewood double decomposition one can write

$$
\begin{aligned}
u v= & \Pi_{u}^{\prime \prime} v+\Pi_{v}^{\prime \prime} u \\
& +\left(\sum_{p \geq 2, p^{\prime} \geq 0} \Delta_{p} S_{p^{\prime}+3}^{\prime} u \cdot S_{p-2} \Delta_{p}^{\prime} v+\sum_{p \geq 2, p^{\prime} \geq 0} S_{p-2} \Delta_{p^{\prime}}^{\prime} u \cdot \Delta_{p} S_{p^{\prime}+3}^{\prime} v\right)
\end{aligned}
$$




$$
\begin{aligned}
& +\left(\sum_{q-2 \leq p \leq q+2, p^{\prime} \geq 2} \Delta_{p p^{\prime}} u \cdot \Delta_{q} S_{p^{\prime}-2}^{\prime} v\right. \\
& \left.+\sum_{q-2 \leq p \leq q+2, p^{\prime} \geq 2} \Delta_{p} S_{p^{\prime}-2}^{\prime} u \cdot \Delta_{q p^{\prime}} v\right) \\
& +\sum_{q-2 \leq p \leq q+2, q^{\prime}-2<p^{\prime}<q^{\prime}+2} \Delta_{p p^{\prime}} u \cdot \Delta_{q q^{\prime}} v,
\end{aligned}
$$

where $\Pi^{\prime \prime}$ denotes the 2-paramultiplication operator

$$
\Pi_{u}^{\prime \prime} v=\sum_{p, p^{\prime} \geq 2} S_{p-2, p^{\prime}-2} u \cdot \Delta_{p p^{\prime}} v .
$$

Now we estimate the norms of each component of the expression for $u v$.

(i) $w_{1}=\Pi_{v}^{\prime \prime} u$. Every component in $w_{1}$ has spectrum (i.e. support of the Fourier transform) in $\left\{2^{p-2} \leq|\xi| \leq 9 \cdot 2^{p-2} ; 2^{p^{\prime}-2} \leq\left|\xi^{\prime}\right| \leq 9 \cdot 2^{p^{\prime}-2}\right\}$ (we use the fact that $F(f \cdot g)=F f * F g$, and $\operatorname{supp} f * g \subset \operatorname{supp} f+\operatorname{supp} g)$. According to the equality $S_{p-2, p^{\prime}-2} v=\sum_{r^{\prime} \leq p} \Delta_{r^{\prime}-3} S_{p^{\prime}-2} v$ and (3),

$$
\begin{aligned}
\left\|S_{p-2, p^{\prime}-2} v \cdot \Delta_{p p^{\prime}} u\right\|_{0} & \leq\left\|S_{p-2, p^{\prime}-2} v\right\|_{L^{\infty}}\left\|\Delta_{p p^{\prime}} u\right\|_{0} \\
& \leq C\left\|\Delta_{p p^{\prime}}\right\|_{0} \leq 2^{-p s-p^{\prime} s^{\prime}} \varepsilon_{p p^{\prime}}
\end{aligned}
$$

with $\left\{\varepsilon_{p p^{\prime}}\right\} \in \ell^{2}\left(\mathbb{N}^{2}\right)$. In the last inequality we have used Theorem 1. From Lemma 1 it follows that $w_{1} \in H^{s, s^{\prime}}$.

(ii) $w_{2}=\sum_{q-2 \leq p \leq q+2, p^{\prime} \geq 2} \Delta_{p p^{\prime}} u \cdot \Delta_{q} S_{p^{\prime}-2}^{\prime} v$ has five components $\sum_{p \geq 1, p^{\prime} \geq 2} \Delta_{p p^{\prime}} u \cdot \Delta_{p+N} S_{p^{\prime}-2}^{\prime} v$, each of them has spectrum $\left\{|\xi| \leq \gamma 2^{p} ; 2^{p^{\prime}-2}\right.$ $\left.\leq\left|\xi^{\prime}\right| \leq 9 \cdot 2^{p^{\prime}-2}\right\}$, and

$$
\left\|\Delta_{p p^{\prime}} u \cdot \Delta_{p+N} S_{p^{\prime}-2}^{\prime} v\right\|_{0} \leq\left\|\Delta_{p p^{\prime}} u\right\|_{0}\left\|\Delta_{p+N} S_{p^{\prime}-2}^{\prime} v\right\|_{L^{\infty}} \leq 2^{-p(s+\varrho)-p^{\prime} s^{\prime}} \varepsilon_{p p^{\prime}}
$$

with $\left\{\varepsilon_{p p^{\prime}}\right\} \in \ell^{2}\left(\mathbb{N}^{2}\right)$. Hence according to Lemma $3, w_{2} \in H^{s+\varrho, s^{\prime}}$.

(iii) $w_{3}=\sum_{q-2 \leq p \leq q+2, q^{\prime}-2<p^{\prime}<q^{\prime}+2} \Delta_{p p^{\prime}} u \cdot \Delta_{q q^{\prime}} v=\sum_{q, q^{\prime} \geq-1} w_{q q^{\prime}}$. The spectrum of $w_{q q^{\prime}}$ lies in the 2-ball $\left\{|\xi| \leq \gamma \cdot 2^{q} ;\left|\xi^{\prime}\right| \leq \gamma \cdot 2^{q^{\prime}}\right\}$. As $s>0$, according to Lemma 2 we have $\sum_{q \text { or } q^{\prime}=-1} w_{q q^{\prime}} \in H^{s, \infty}$. Here we have used the fact that $\left\|S_{0} \Delta_{q} v\right\|_{L^{\infty}} \leq c\left\|\Delta_{q} v\right\|_{L^{\infty}} \leq C$. For $q, q^{\prime} \geq 0$ we have

$$
\left\|w_{q q^{\prime}}\right\|_{L^{1}} \leq\left(\sum_{q-2 \leq p \leq q+2, q^{\prime}-1 \leq p^{\prime} \leq q^{\prime}+2}\left\|\Delta_{p p^{\prime}} u\right\|_{0}\right)\left\|\Delta_{q q^{\prime}} v\right\|_{0} \leq 2^{-2 q s-2 q^{\prime} s^{\prime}} \varepsilon_{q q^{\prime}}
$$

with $\left\{\varepsilon_{q q^{\prime}}\right\} \in \ell^{2}\left(\mathbb{N}^{2}\right)$. The operator $\Delta_{k^{\prime}}^{\prime}: L^{1} \rightarrow L^{1}$ is bounded (with norm 
independent of $\left.k^{\prime}\right), s>(n-k) / 2$ and $q^{\prime} \leq q+1$, hence for $k^{\prime} \geq-1$,

$$
\begin{aligned}
\left\|\Delta_{k^{\prime}}^{\prime} \sum_{q^{\prime} \geq 0} w_{q q^{\prime}}\right\|_{L^{1}} & \leq C \sum_{k^{\prime} \leq q^{\prime}+N}\left\|w_{q q^{\prime}}\right\|_{L^{1}} \leq C \sum_{k^{\prime} \leq q^{\prime}+N} 2^{-2 q s-2 q^{\prime} s^{\prime}} \varepsilon_{q q^{\prime}} \\
& \leq C(s, k, n) \cdot 2^{-q(s+(n-k) / 2)} \sum_{k^{\prime} \leq q^{\prime}+N} 2^{-q\left(s+2 s^{\prime}(n-k) / 2\right)} \varepsilon_{q q^{\prime}}
\end{aligned}
$$

with $\left\{\varepsilon_{q q^{\prime}}\right\} \in \ell^{2}\left(\mathbb{N}^{2}\right)$. If additionally $s+2 s^{\prime}-(n-k) / 2>0$, then

$$
\left\|\Delta_{k^{\prime}}^{\prime} \sum_{q^{\prime} \geq 0} w_{q q^{\prime}}\right\|_{L^{1}} \leq 2^{-q(s+(n-k) / 2)-k^{\prime}\left(s+2 s^{\prime}-(n-k) / 2\right)} \varepsilon_{q, k^{\prime}+1}
$$

with $\left\{\varepsilon_{q k^{\prime}}\right\} \in \ell^{2}\left(\mathbb{N}^{2}\right)$, and

$$
\begin{aligned}
\left\|\Delta_{k^{\prime}}^{\prime} \sum_{q^{\prime} \geq 0} w_{q q^{\prime}}\right\|_{0} & \leq C \cdot 2^{q(n-k) / 2+k^{\prime} k / 2}\left\|\Delta_{k^{\prime}}^{\prime} \sum_{q^{\prime} \geq 0} w_{q q^{\prime}}\right\|_{L^{1}} \\
& \leq 2^{-q s-k^{\prime}\left(s+2 s^{\prime}-n / 2\right)} \varepsilon_{q, k^{\prime}+1}
\end{aligned}
$$

with $\left\{\varepsilon_{q k^{\prime}}\right\} \in \ell^{2}\left(\mathbb{N}^{2}\right)$. Here we have used the inequality $k^{\prime} \leq q^{\prime}+N$, and the equality $F \Delta_{k^{\prime}}^{\prime} w_{q q^{\prime}}=\psi^{\prime}\left(2^{-k^{\prime}} \xi^{\prime}\right) F w_{q q^{\prime}}=\psi^{\prime}\left(2^{-k^{\prime}} \xi^{\prime}\right) \eta\left(2^{-q} \xi^{\prime \prime}\right) F w_{q q^{\prime}}$ where $\eta \in C_{0}^{\infty}$ and $\eta\left(2^{-q \xi^{\prime \prime}}\right)=1$ if $\xi$ belongs to supp $F w_{q q^{\prime}}$.

The above calculations and the lemmas yield

$$
w_{3}=\sum_{q \geq 0, k^{\prime} \geq-1} \Delta_{k^{\prime}}^{\prime} \sum_{q^{\prime} \geq 0} w_{q q^{\prime}} \in H^{s, s+2 s^{\prime}-n / 2} .
$$

(iv) $w_{4}=\sum_{p \geq 2, p^{\prime} \geq 0} \Delta_{p} S_{p^{\prime}+3}^{\prime} u \cdot S_{p-2} \Delta_{p^{\prime}}^{\prime} v=\sum w_{p p^{\prime}}$. Now supp $F u_{p p^{\prime}}$ lies in the set $\left\{2^{p-2} \leq|\xi| \leq 9 \cdot 2^{p-2},\left|\xi^{\prime}\right| \leq 5 \cdot 2^{p^{\prime}+1}\right\}$. Let $F_{x^{\prime}}$ denote the Fourier transform with respect $x^{\prime}$. Then

$$
\begin{aligned}
F_{x^{\prime}} \Delta_{p} S_{p^{\prime}+3}^{\prime} u & =\phi^{\prime}\left(2^{-p^{\prime}-3} \xi^{\prime}\right) F_{x^{\prime}} \Delta_{p} u=\sum_{q^{\prime} \leq p^{\prime}+2} \psi^{\prime}\left(2^{-q^{\prime}} \xi^{\prime}\right) F_{x^{\prime}} \Delta_{p} u \\
& =\left(\sum_{q^{\prime} \leq p^{\prime}+2} \psi^{\prime}\left(2^{-q^{\prime}} \xi^{\prime}\right)\right)\left(\sum_{r^{\prime} \leq p^{\prime}+2} \psi^{\prime}\left(2^{-r^{\prime}} \xi^{\prime}\right)\right) F_{x^{\prime}} \Delta_{p} u \\
& =\sum_{q^{\prime} \leq p^{\prime}+2, r^{\prime} \leq p^{\prime}+2} \psi^{\prime}\left(2^{-q^{\prime}} \xi^{\prime \prime}\right) F_{x^{\prime}} \Delta_{p r^{\prime}} u .
\end{aligned}
$$

Hence

$$
\begin{aligned}
\left|\Delta_{p} S_{p^{\prime}+3}^{\prime} u\right| & \leq C \sum_{q^{\prime} \leq p^{\prime}+2} 2^{q^{\prime} k} \psi^{\prime}\left(2^{q^{\prime} k} x^{\prime}\right) *\left|\Delta_{p q^{\prime}} u\right| \\
& \leq C \sum_{q^{\prime} \leq p^{\prime}+2}\left\|2^{q^{\prime} k} \psi^{\prime}\left(2^{q^{\prime} k} x^{\prime}\right)\right\|_{L_{x^{\prime}}^{2}}\left\|\Delta_{p p^{\prime}} u\right\|_{L_{x^{\prime}}^{2}} \\
& \leq C \sum_{q^{\prime} \leq p^{\prime}+2} 2^{q^{\prime} k / 2}\left\|\Delta_{p p^{\prime}} u\right\|_{L_{x^{\prime}}^{2}},
\end{aligned}
$$




$$
\left\|\Delta_{p} S_{p^{\prime}+3}^{\prime} u\right\|_{L_{x^{\prime \prime}}^{2}\left(L_{x^{\prime}}^{\infty}\right)} \leq C \sum_{q^{\prime} \leq p^{\prime}+2} 2^{q^{\prime} k / 2}\left\|\Delta_{p q^{\prime}} u\right\|_{0}
$$

and

$$
\left\|\Delta_{p} S_{p^{\prime}+3}^{\prime} u\right\|_{L_{x^{\prime \prime}}^{2}\left(L_{x^{\prime}}^{\infty}\right)} \leq \begin{cases}2^{-p s-p^{\prime}\left(s^{\prime}-k / 2\right)} \varepsilon_{p p^{\prime}} & \text { if } s^{\prime}<k / 2, \\ 2^{-p s+p^{\prime} \varepsilon} \varepsilon_{p p^{\prime}} & \text { if } s^{\prime}=k / 2, \\ 2^{-p s} \varepsilon_{p} & \text { if } s^{\prime}>k / 2,\end{cases}
$$

with $\varepsilon>0,\left\{\varepsilon_{p p^{\prime}}\right\} \in \ell^{2}\left(\mathbb{N}^{2}\right)$, and $\left\{\varepsilon_{p}\right\} \in \ell^{2}(\mathbb{N})$.

Similarly (this time using the Fourier transform $F_{x^{\prime \prime}}$ ) we get

$$
\begin{aligned}
\left\|S_{p-2} \Delta_{p^{\prime}}^{\prime} v\right\|_{L_{x^{\prime \prime}}^{\infty}\left(L_{x^{\prime}}^{2}\right)}^{\prime} & \leq \sum_{q \leq p-1, q \geq p^{\prime}-1} 2^{-q(s-(n-k) / 2)-p^{\prime} s^{\prime}} \varepsilon_{q p^{\prime}} \leq 2^{-p^{\prime}\left(s+s^{\prime}-(n-k) / 2\right)} \varepsilon_{p^{\prime}}
\end{aligned}
$$

with $\left\{\varepsilon_{p^{\prime}}\right\} \in \ell^{2}(\mathbb{N})$. Hence

$$
\left\|\Delta_{p} S_{p^{\prime}+3}^{\prime} u \cdot S_{p-2} \Delta_{p^{\prime}}^{\prime} v\right\|_{0} \leq 2^{-p s-p^{\prime}\left(s^{\prime}+p\right)} \varepsilon_{p p^{\prime}}
$$

with $\left\{\varepsilon_{p p^{\prime}}\right\} \in \ell^{2}\left(\mathbb{N}^{2}\right)$. According to our lemmas $w_{4} \in H^{s, s^{\prime}+\varrho}$ if $s^{\prime}+\varrho>0$. For $s+\varrho<0$ (i.e. $s^{\prime}<0$ ) we proceed a little differently:

$$
\begin{aligned}
\left\|\Delta_{p} S_{p^{\prime}+3}^{\prime} u \cdot S_{p-2} \Delta_{p^{\prime}}^{\prime} v\right\|_{L_{x^{\prime \prime}}^{2}\left(L_{x^{\prime}}^{1}\right)} & \leq\left\|\Delta_{p} S_{p^{\prime}+3}^{\prime} u\right\|_{0} \cdot\left\|S_{p-2} \Delta_{p^{\prime}}^{\prime} v\right\|_{L_{x^{\prime \prime}}^{\infty}\left(L_{x^{\prime}}^{2}\right)} \\
& \leq 2^{-p s-p^{\prime}\left(s+2 s^{\prime}-(n-k) / 2\right)} \varepsilon_{p p^{\prime}}
\end{aligned}
$$

with $\left\{\varepsilon_{p p^{\prime}}\right\} \in \ell^{2}\left(\mathbb{N}^{2}\right)$. Since $s+2 s^{\prime}-(n-k) / 2>0$, for $r^{\prime} \geq-1$ we have

$$
\left\|\Delta_{r^{\prime}}^{\prime} \sum_{p^{\prime}} \Delta_{p} S_{p^{\prime}+3}^{\prime} u \cdot S_{p-2} \Delta_{p^{\prime}}^{\prime} v\right\|_{L_{x^{\prime \prime}}^{2}\left(L_{x^{\prime}}^{1}\right)} \leq 2^{-p s-r^{\prime}\left(s+2 s^{\prime}-(n-k) / 2\right)} \varepsilon_{p, r+1^{\prime}} .
$$

The operator $\Delta_{r^{\prime}}^{\prime}: L_{x^{\prime \prime}}^{2}\left(L_{x^{\prime}}^{1}\right) \rightarrow L_{x^{\prime \prime}}^{2}\left(L_{x^{\prime}}^{1}\right)$ is bounded (with norm independent of $r^{\prime}$ ).

Proceeding as before we get

$$
\left\|\Delta_{r^{\prime}}^{\prime} \sum_{p^{\prime}} \Delta_{p} S_{p^{\prime}+3}^{\prime} u \cdot S_{p-2} \Delta_{p^{\prime}}^{\prime} v\right\|_{0} \leq 2^{-p s-r^{\prime}\left(s+2 s^{\prime}-(n-k) / 2\right)} \varepsilon_{p, r+1^{\prime}}
$$

and

$$
w_{4}=\sum_{p, r^{\prime}} \Delta_{r^{\prime}}^{\prime} \sum_{p^{\prime}} \Delta_{p} S_{p^{\prime}+3}^{\prime} u \cdot S_{p-2} \Delta_{p^{\prime}}^{\prime} v \in H^{s, s+2 s^{\prime}-n / 2} .
$$

Hence we have proved the following theorem:

Theorem 3. If $s>(n-k) / 2, s+s^{\prime}>n / 2$ and $s+2 s^{\prime}>(n-k) / 2$, then the space $H^{s, s^{\prime}}$ is an algebra over $\mathbb{C}$ under pointwise multiplication.

Similarly to [1] we can also prove

Theorem 4. Let $u \in H^{s, s^{\prime}}, s>(n-k) / 2, s+s^{\prime}>n / 2$. The operator

$$
\Pi_{u}^{\prime}: H^{t, t^{\prime}} \rightarrow H^{t, t^{\prime}}, \quad-s<t \leq s,
$$


is bounded with norm bounded by $c\|u\|_{s, s^{\prime}}$. Moreover, the operator

$$
\Pi_{u}^{\prime}-\Pi_{u}^{\prime \prime}: H^{t, t^{\prime}} \rightarrow H^{t, t^{\prime}+\varrho}
$$

is continuous, where $-s \leq t \leq s, \varrho>0, \varrho=\min \left(s-(n-k) / 2, s+s^{\prime}-n / 2\right)$ if $s^{\prime} \neq k / 2$, and $\varrho<s-(n-k) / 2$ if $s^{\prime}=k / 2$.

\section{References}

[1] M. Sable-Tougeron, Régularité microlocale pour des problèmes aux limites non linéaires, Ann. Inst. Fourier (Grenoble) 36 (1986), no. 1, 39-82.

[2] B. Yu. Sternin, Elliptic and parabolic boundary value problems on manifolds with boundary components of different dimensions, Trudy Moskov. Mat. Obshch. 15 (1966), 346-382 (in Russian).

Institute of Mathematics

Warsaw University

Banacha 2

02-097 Warszawa, Poland

E-mail: jhk@mimuw.edu.pl 